IdeAs

Idées d'Amériques

Poètes et éditeurs : diffuser la poésie d'avant-garde américaine (depuis 1945)

\title{
Les droits des victimes dans les accords de paix de
} La Havane

Diana Gómez

(2) OpenEdition

Journals

Édition électronique

URL : https://journals.openedition.org/ideas/1846

DOI : $10.4000 /$ ideas. 1846

ISSN : 1950-5701

Éditeur

Institut des Amériques

Référence électronique

Diana Gómez, « Les droits des victimes dans les accords de paix de La Havane », IdeAs [En ligne], 9 |

2017, mis en ligne le 03 juillet 2017, consulté le 20 octobre 2022. URL : http://

journals.openedition.org/ideas/1846 ; DOI : https://doi.org/10.4000/ideas.1846

Ce document a été généré automatiquement le 20 octobre 2022

\section{(2) $\odot \Theta \Theta$}

Creative Commons - Attribution - Pas d'Utilisation Commerciale - Pas de Modification 4.0 International - CC BY-NC-ND 4.0

https://creativecommons.org/licenses/by-nc-nd/4.0/ 


\title{
Les droits des victimes dans les accords de paix de La Havane
}

\author{
Diana Gómez
}

Texte traduit de l'espagnol par Camille Boutron

1 Pendant les quatre ans qu'ont duré les négociations de paix entre les FARC et le gouvernement colombien, la catégorie de victime a signifié un lieu de dispute et d'énonciation qui continue de représenter un terrain contentieux à l'heure actuelle. Ce texte cherche ainsi à s'interroger sur le sens et les trajectoires de la catégorie de victime dans le cadre du processus de paix, ainsi que sur les enjeux posés par la mise en application de l'accord en relation aux droits des sujets victimisés.

\section{La constitution de lieux pour nommer et se nommer}

2 Les catégories apparaissent comme des tentatives de comprendre et donner du sens à la réalité. Elles représentent à la fois des efforts de différenciation et de généralisation qui peuvent parfois donner lieu à la construction d'appréciations finalement peu pertinentes vis-à-vis de la complexité de la réalité. En tant que catégorie, la notion de « victimes " n'est pas figée. En Colombie, elle commence à émerger dans les années 1990 grâce au travail des organisations de droits humains, à l'effort de mouvements pacifistes ou féministes pour avancer vers une sortie négociée du conflit armé, ou encore grâce à la circulation de discours sur les droits humains au sein des institutions publiques colombiennes.

Pendant les années 1980, ce sont avant tout les proches de personnes détenues et disparues qui composent les associations de victimes. Malgré une histoire de lutte et de dénonciation permanentes de ces organisations, la société colombienne au début des années 2000 ne reconnaissait comme victimes valides et existantes que celles des guérillas et groupes paramilitaires. Ces victimes ont été dotées d'un statut légal grâce à la loi Justice et Paix, à travers laquelle s'est réalisé le processus de démobilisation des groupes paramilitaires. Or, si l'Etat reconnaît ce type de victimes, il continue de nier les 
autres sujets victimisés dont il est responsable. Cela a contribué à constituer, et ce depuis les années 1970, un traitement asymétrique des victimes en Colombie.

4 Il n'est pas fortuit que ce soit dans le contexte de la démobilisation des groupes paramilitaires en 2005 que surgit le Mouvement des Victimes de Crimes d'Etat (MOVICE). Le MOVICE se présente ainsi comme un produit de la lutte de diverses organisations sociales qui cherchent à rendre visible la violence systématique contre les leaders sociaux issus des partis et mouvements sociaux de gauche. L'émergence de ces organisations a permis de rendre publiques les victimes de crimes d'Etat, en rupture avec l'invisibilisation permanente dont elles étaient l'objet. Elles se sont ainsi converties en acteurs centraux de la lutte pour la mémoire, la vérité, la justice, la réparation et la non répétition, interpellant le processus de Justice et Paix et ouvrant de nouveau le débat sur l'existence de la violence socio-politique en Colombie.

\section{Toutes les victimes occupent-elles une place centrale dans les accords de La Havane?}

5 L'agenda des négociations pactées entre le gouvernement et les FARC est complexe. Cependant, le cinquième point concernant les victimes possède une dimension particulière dans la mesure où, en plus de matérialiser les droits des sujets victimisés, il définit le type et la durée des sanctions qui doivent être appliquées aux responsables qui doivent, en outre, s'en expliquer.

Or reconnaître l'existence des victimes implique en même temps reconnaître les victimaires et poser la question du degré de responsabilité de chacune des parties engagées dans la négociation. Dans ce cadre, la catégorie de victime représente un objet de controverse, et tant l'Etat que la guérilla des FARC ont en effet cherché à nier leur responsabilité dans les violations de droits humains en essayant de se revendiquer euxmêmes comme victimes. Cela a dès lors ouvert des questionnements sur la pertinence d'effacer ou au contraire de maintenir les frontières entre combattants et civils, ou encore sur le statut même de victime. Ces interrogations ne sont pas seulement ontologiques mais ont une conséquence sur la façon dont est comprise la violence, la responsabilité des acteurs et les raisons de leurs actes ou encore les sanctions sociales et judiciaires qui doivent être appliquées.

7 Au milieu de ces confrontations, les sujets victimisés se disputent le droit d'être réellement « centraux » au sein de l'Accord. Cela a signifié leur contribution active au travers des canaux ouverts à la participation des Colombiens aux négociations. La centralité des victimes s'est ainsi manifestée dans la réalisation de forums publics organisés dans le cadre de l'agenda des négociations qui se sont ainsi parfois convertis en scènes de confrontation voilée ou explicite entre les victimes des FARC et l'Etat. D'autre part, 60 victimes ont fait partie de 5 délégations ayant fait le déplacement jusqu'à La Havane pour établir un dialogue plus direct avec la Table de négociation. Certaines «victimes » ayant participé à ces rencontres évoquent ainsi une expérience bénéfique, de rencontre avec leur histoire, révélatrice de l'importance que cela peut avoir de se sentir vues et écoutées, voire interpellées par les responsables de leurs histoires de douleurs. D'autres sujets victimisés se sont retrouvés en revanche face à un format d'expression limité, ce qui les a obligés à raconter leur histoire individuellement, prêtant peu ou pas d'attention à la dimension collective des dommages causés aux communautés indigènes, afro-descendantes, paysannes, de 
femmes, de personnes LGBTI, de partis ou encore de leaders sociaux. Par ailleurs, si les victimes des guérillas ont pu établir un face-à-face avec leurs victimaires, dans le cas des victimes d'Etat la rencontre avec les institutions gouvernementales est restée abstraite, rendant significative la différence entre les victimes selon le responsable présumé.

8 Malgré un langage collectif et quotidien sur la centralité des victimes, la société colombienne, l'Etat, les médias et la Table de négociation n'ont pas tous eu la même approche du concept. La majorité de la société colombienne a considéré en effet que les victimes qui devaient être présentes à La Havane étaient celles de la guérilla. Pour beaucoup de Colombien-nes, il n'y avait aucune raison pour que les victimes d'Etat revendiquent leurs droits et jouent un rôle central au sein du processus de négociation. De fait, si le terme de « victimes » est évoqué en termes généraux et abstraits, lorsqu'il s'agit de lui donner un visage, la majorité du pays voit celui de victime des FARC.

9 Une notion hégémonique de "victime ", générale et abstraite s'est consolidée tout au long du processus de paix. Cela a eu pour effet l'effacement des différences existantes entre les sujets victimisés. Or si toutes les victimes méritent la même reconnaissance, dans un pays comme la Colombie il reste important de prendre en compte les particularités de chacun. Ces différences se déclinent non seulement en relation aux présumés responsables, mais aussi à la forme de reconnaissance donnée par l'Etat et la société, et à la facilitation ou obstruction des tentatives de recherche de vérité et de justice.

10 Effacer la particularité de la victime du crime d'Etat autorise le maintien de la version négationniste de la violence étatique, de ses dimensions et de ses conséquences mais aussi du rôle de l'État dans le conflit. L'usage abstrait de la catégorie de victime dépossède les sujets victimisés de leur nature politique. Au travers de cette catégorie et de politiques publiques comme la Loi sur les victimes et la restitution des terres, l'Etat a cherché à neutraliser la capacité critique de ces sujets au travers de politiques assistancialistes qui, au lieu de permettre la constitution de sujets délibératifs, tend à les convertir en clients de l'Etat et reproduire ainsi la politique traditionnelle. D'un autre côté, la méconnaissance de la criminalité étatique annule le caractère profondément politique des victimes de ce type de violence, leaders sociaux de gauche, indigènes, afro-descendants ou paysans pour la plupart d'entre eux, qui défendent un modèle de société différent de celui imposé par les élites au pouvoir. De cette manière, et ce malgré le fait que les victimes d'Etat semblent gagner une visibilité et maintenir le positionnement qu'elles avaient adopté pendant le processus de démobilisation des groupes paramilitaires, cette visibilité est devenue instrumentale au milieu du processus de paix, plus médiatique et conjoncturelle que réelle, à tel point qu'elle semble presque avoir régressé depuis les années 1980 et la victoire du «non» au plébiscite du 2 octobre.

11 Ce recul dans la reconnaissance des victimes de crimes d'Etat et la persistance d'un traitement asymétrique se perçoit différemment depuis la victoire du «non» au plébiscite du 2 octobre dernier. En premier lieu, en raison des modifications substantielles de l'Accord dans sa version finale, qui protège les forces armées et la chaîne de commandement. Mais aussi parce que des changements significatifs ont été introduits dans le cadre de la Juridiction spéciale pour la paix (JSP), qui permettent aux militaires de haut rang de ne pas être tenus responsables des crimes commis par leurs subordonnés. Ces changements restreignent la possibilité d'enquêter, de juger et de 
sanctionner la participation directe et indirecte de tiers dans le financement des groupes paramilitaires, et ce malgré le fait qu'ait été établie leur participation dans des crimes de guerre et contre l'humanité, ainsi que l'examen des rapports présentés par les organisations de victimes et de droits humains considérés comme preuve. On a pu par ailleurs observer une certaine mise sous silence de la voix des victimes de crimes d'Etat au sein des espaces de discussion sur le processus de paix et les modifications à apporter à l'accord après la victoire du «non ». Par conséquent, ces victimes ont été exclues des processus de définition finale et de présentation des mécanismes de justice transitionnelle au cours du Fast Track ${ }^{1}$ (Juridiction spéciale pour la paix et Commission Vérité). Finalement, on assiste à l'heure actuelle à un processus de re-légitimation de l'institution militaire qui fait l'impasse sur son rôle actif dans le conflit armé. Cela a récemment été au centre d'une controverse suscitée par la nomination de représentants du ministère de la Défense au sein du Conseil de direction du Centre national de mémoire historique ${ }^{2}$.

\section{Les victimes face aux enjeux de la mise en application de l'accord de paix}

12 Les défis posés par l'application des accords de La Havane relatifs aux droits des victimes sont nombreux. Il est nécessaire que l'ensemble du pays reconnaisse la douleur et la souffrance que des millions de personnes ont endurées en conséquence d'un usage de la violence comme stratégie politique. Par ailleurs, la société colombienne doit reconnaitre l'apport des victimes s'étant mobilisées depuis la sphère civile et la pratique démocratique pour la construction de la paix. Finalement, il est urgent de faire respecter le droit des victimes de façon à ce qu'elles puissent commencer un processus de "guérison " et qu'elles soient traitées, ainsi que leurs proches, comme des sujets de droits.

13 La matérialisation de leurs droits passe par une posture qui cherche à éliminer les causes structurelles de la violence. Pour cela il est nécessaire d'aller au-delà du modèle standard et hégémonique de justice transitionnelle, qui dans d'autres contextes s'est montré incapable de satisfaire les attentes des sujets victimisés et d'inspirer une transformation de la société. En ce sens, l'accord de La Havane est insuffisant. Satisfaire les droits des victimes nécessite de répondre à l'ensemble des univers des sujets victimisés dans une période réduite ( 3 ans). D'un autre côté, les 10 ans de mandat octroyés à JSP font courir le risque d'une dissipation de la justice et de maintien de l'impunité structurelle qui caractérise le pays.

Un autre enjeu concerne le traitement symétrique des victimes. Cela suppose de garantir les mêmes droits depuis les réalités et mesures particulières de chaque individu. Jusqu'à présent, le Fast Track ne s'est pas révélé très positif pour les victimes de crimes d'Etat. Il n'est en outre pas de très bon augure qu'avant même qu'elle ait été mise en place, certains membres des forces de l'ordre incarcérés pour violations de droits humains saisissent à leur profit la JSP afin d'obtenir leur liberté conditionnelle, et ce grâce à un récent décret des ministères de la Défense et de la Justice ${ }^{3}$. La Colombie a besoin d'un réel débat public sur la valeur de la justice et la sanction sociale.

Les mécanismes prévus à La Havane pour répondre aux droits des victimes, enfin, font face à d'importants défis. Dans le cadre de la future Commission Vérité, il est urgent 
que l'Etat procède à la déclassification des archives d'intelligence militaire, dans la mesure où elles contiennent des informations susceptibles d'apporter la lumière sur les crimes perpétrés par les forces de l'ordre. Il est également nécessaire que les FARC respectent leur engagement à ouvrir leurs propres archives et rendre publiques les informations relatives à leurs actions.

Etant donné la position privilégiée obtenue par les militaires dans la JSP, des secteurs de la droite colombienne, les militaires et l'Etat lui-même semblent peu soucieux d'une véritable clarification des faits. Or les tensions entre justice et vérité tendent à se multiplier, rendant essentielle la constitution d'une Commission Vérité capable de rendre compte, au-delà des perspectives biaisées de chacun des acteurs, de la complexité des faits ayant marqué l'histoire du conflit armé. Cela implique l'identification des responsables, la compréhension des modalités de la violence et l'évaluation de ses impacts. La Colombie fait face à la nécessité d'une récupération de son histoire qui permette à ses ressortissants de se réapproprier leur mémoire et de se constituer en sujets critiques ouverts au changement social.

Dans un tel scénario, la catégorie de "victimes » ne doit pas être mobilisée pour penser la réalité du pays depuis une perspective dichotomique: victimes vs. victimaires, gentils vs. méchants, ou encore guérilla vs Etat. Elle n'est pas nécessaire non plus pour gagner des prix comme le Nobel de la Paix. La catégorie de victimes doit permettre de prêter une attention collective aux conditions structurelles à l'origine de la violence et qui ont à voir avec les exclusions sociales, politiques, économiques, mais aussi culturelles et ontologiques. En ce sens, la vérité et la justice doivent être comprises comme des droits collectifs, de la société dans son ensemble.

\section{NOTES}

1. La procédure de Fast Track (ou "voie rapide") a été adoptée à la suite de la signature finale et de l'accord de paix et de sa validation par le Congrès colombien fin 2016 pour pouvoir rapidement mettre en place les premières mesures de sa mise en application, notamment dans le cadre de la justice transitionnelle.

2. http://colombia2020.elespectador.com/politica/polemica-por-el-cupo-de-los-militares-en-elcentro-de-memoria-historica

3. http://www.elespectador.com/noticias/judicial/preocupacion-por-beneficios-de-la-jep-paramilitares-articulo-692630 


\section{AUTEUR}

DIANA GÓMEZ

Professeure assistante, CIDER, Universidad de los Andes, membre du MOVICE et de Hijos e Hijas por la Memoria y en contra de la Impunidad 